\title{
Research on optimal takeoff positions of UAV integrated GNSS - RTK in producing large scale topological maps for open - pit mines
}

\author{
Canh Van Le *, Cuong Xuan Cao, Ha Thu Thi Le \\ Faculty of Geomatics and Land Administration, Hanoi University of Mining and Geology, Vietnam
}

\begin{abstract}
ARTICLE INFO
ABSTRACT

Article history:

Received $30^{\text {th }}$ June 2020

Revised 23 $3^{\text {rd }}$ July 2020

Accepted $31^{\text {st }}$ Oct. 2020

Keywords:

DSM,

Flight height,

Open - pit mines,

Taking off location,

UAV/RTK.

Unmanned aerial vehicles (UAV) are widely used for establishing large scale topological maps. Recently, drones have been integrated with highquality GNSS receivers which allows real time kinematic positioning (RTK), so are called UAV/RTK. This technology is beneficial to surveyors as they do not need to establish many ground control points in mapping such a complex terrain as open-pit mines. DJI Phantom 4 RTK (P4K) is a $U A V / R T K$ which is of much interest due to its small size and low cost. For open-pit mines, the takeoff position of P4K needs to be seriously considered because of its influence on the accuracy of the digital surface model (DSM) and safety of survey flights. This article presents the method of determining the optimal takeoff positions for UAV in large scale mapping for open pit mines. To evaluate this method, a site of steep and rugged terrain with an area of 80 hectares at the Coc Sau coal mine was chosen as the study area. The results indicate that two optimal locations with altitudes of $+50 \mathrm{~m}$ and $+160 \mathrm{~m}$ could be used for taking off the P4K. The accuracy of DSM generated from UAV images using the optimal positions satisfied the accuracy requirement of large scale topological maps at the deepest area of the mine (the altitude of $-60 \mathrm{~m}$ ).
\end{abstract}

Copyright (C) 2020 Hanoi University of Mining and Geology. All rights reserved.

${ }^{*}$ Corresponding author

E - mail: levancanh@humg.edu.vn

DOI: 10.46326/JMES.2020.61(5).06 


\title{
Nghiên cứu lựa chọn vị trí cất cánh cho thiết bị bay không người lái tích hợp GNSS động phục vụ đo vẽ thành lập bản đồ địa hình tỷ lệ lớn cho các mỏ lộ thiên
}

\author{
Lê Văn Cảnh *, Cao Xuân Cường, Lê Thị Thu Hà \\ Khoa Trắc địa - Bản đồ và Quản lý đất đai , Trường Đại học Mỏ - Địa chất, Việt Nam
}

THÔNG TIN BÀI BÁO TÓM TẮT

Quá trình:

Nhận bài 30/06/2020

Sửa xong 23/7/2020

Chấp nhận đăng 31/10/2020

Tù̀ khóa:

Chiều cao bay chụp,

DSM,

Mỏ lộ thiên,

UAV/RTK,

Vị trí cất cánh.
Thiết bị bay không người lái (UAV) đang được úng dụng rộng rãi trong công tác đo vẽ thành lập bản đồ địa hình. Gần đây, UAV được tích hợp thêm thiết bị định vị tâm ảnh bằng công nghệ GNSS động (UAV/RTK) đã giúp tăng cường khả năng đo vẽ thành lập bản đồ địa hình bằng công nghề này. Hiện nay, trong thưc tế sản xuất, DJI Phantom 4 RTK (P4K) là UAV/RTK được quan tâm nhiều do thiết bị này nhỏ gọn, giá thành thấp. Đối với các địa hình có chênh cao thay đổi lớn như các mó lộ thiên thì việc xác định được vị trí cất cánh của máy bay sẽ quyết định đến độ chính xác của mô hình số bề mặt (DSM) và an toàn của hoạt động bay chụp. Bài báo này sẽ giới thiệu phương pháp xác định vị trí cất cánh phù hợp cho P4K khi bay chụp phục vụ đo vẽ bản đồ địa hình tỷ lệ lớn cho mỏ lộ thiên. Để thực hiện mục tiêu này, tại mỏ than Cọc Sáu, khu vực rộng khoảng 80 ha có chênh cao địa hình lớn ( $(300 m)$, được khảo sát để xác định vị trí cất cánh cho P4K. Máy bay được chọn cất cánh tại hai vị trí có độ cao $+50 \mathrm{~m}$ và $+160 \mathrm{~m}$, với vị trí sâu nhất của đia hình mỏ (- $60 \mathrm{~m}$ ) vẫn đảm bảo độ chính xác thành lập bản đồ địa hình tỷ lệ lớn theo qui pham trắc địa mỏ. DSM được thành lập tù ảnh bay chup P4K kết hợp với 02 điểm khống chế ảnh mặt đất có độ chính xác ở mức độ $\mathrm{cm}$.

C 2020 Trường Đại học Mỏ - Địa chất. Tất cả các quyền được bảo đảm.

\section{Mở đầu}

Bản đồ địa hình là tài liệu quan trọng phục vụ cho công tác lập kế hoạch khai thác, tính toán khối lượng xúc bốc (Nguyễn Đình Bé và nnk., 1998). Đo vẽ chi tiết thành lập bản đồ địa hình là công tác được thực hiện thường xuyên tại các mỏ lộ thiên

*Tác giả liên hệ

E - mail: levancanh@humg.edu.vn DOI: 10.46326/JMES.2020.61(5).06
(Võ Chí Mỹ, 2016). Theo qui phạm trắc địa mỏ, bản đồ đo vẽ cập nhật định kỳ ở mỏ lộ thiên thành lập ở tỷ lệ $1 / 1000$ đối với công trường cơ giới và 1/500 đối với công trường thủ công (Tập đoàn Công nghiệp Than - Khoáng sản Việt Nam, 2015).

Hiện nay, công tác đo vẽ chi tiết thành lập bản đồ tại các mỏ lộ thiên chủ yếu sử dụng máy toàn đạc điện tử (Lê Văn Cảnh và nnk., 2020). Tuy nhiên, phương pháp này được đánh giá là có chi phí cao, tốn nhiều thời gian và công sức; gặp khó khăn khi thực hiện trong điều kiện địa hình và môi trường phức tạp, có thể gây mất an toàn lao động 
(Bùi Tiến Diệu và nnk., 2016; Nguyễn Quốc Long và Lê Văn Cảnh, 2020), và ngày càng khó khăn hơn khi các mỏ lộ thiên khai thác xuống sâu, với chênh cao địa hình và các góc dốc bờ tầng ngày càng lớn.

Gần đây, các thiết bị bay không người lái (UAV) đã được ứng dụng trong công tác đo vẽ thành lập mô hình số bề mặt, bản đồ địa hình mỏ lộ thiên (Dieu Tien Bui và nnk., 2017; Lee và Choi, 2015; Nguyen Quoc Long và nnk., 2019). Các tác giả đã chỉ ra rằng công nghệ UAV hoàn toàn đáp ứng được yêu cầu về độ chính xác, giảm thời gian và sức lao động so với phương pháp đo đạc truyền thống tại các mỏ. Gần đây, việc tích hợp công nghệ đo GNSS động lên thiết bị UAV (UAV/RTK) được biết đến như một giải pháp nâng cao độ chính xác định vị tâm ảnh khi bay chụp và thay thế cho các điểm khống chế ảnh mặt đất (Dinkov \& Kitev, 2020). Thiết bị bay không người lái có tích hợp RTK (Real time kinematic - đo động thời gian thực) mang lại sự hiệu quả và cơ động trong lĩnh vực đo đạc địa hình chính là Phantom 4 RTK (P4K), đây là loại máy bay nhỏ gọn bay ở độ cao thấp của hãng DJI. Tuy nhiên, để đạt được độ chính xác theo yêu cầu, cần thiết phải thiết kế được chiều cao bay chụp phù hợp.

Việc tính toán chiều cao bay chụp cho máy bay có gắn máy ảnh phổ thông như Phantom 4 Pro, $\mathrm{P} 4 \mathrm{~K}$ được nhắc đến trong nghiên cứu của tác giả Jacobsen (Jacobsen, 2005). Trong nghiên cứu này, tác giả đã tính toán được độ phân giải ảnh mặt đất cần thiết phù hợp với tỷ lệ bản đồ cần thành lập (Jacobsen, 2005). Tác giả Trần Trung Anh và nnk. (2019) đã đưa ra được hệ số tương quan giữa sai số trung phương vị trí điểm trên mô hình số địa hình (DSM) với độ phân giải ảnh mặt đất (GSD). Trong một nghiên cứu khác, Jing He và cộng sự đã tính toán độ cao bay chụp cho máy bay, mức độ phủ trùm ảnh và GSD cần đạt được (He và nnk., 2012). Tuy nhiên, đối với địa hình phức tạp, chênh cao và góc dốc địa hình lớn như ở mỏ lộ thiên, để đạt được GSD theo yêu cầu và chiều cao bay chụp như trong các nghiên cứu trên, cần phải lựa chọn vị trí cất cánh phù hợp cho thiết bi UAV. Để đảm bảo hai yêu tố là an toàn cho máy bay, đạt GSD theo yêu cầu, đồng thời đảm bảo yếu tố kinh tế. Trên thực tế, chưa có nghiên cứu nào về lựa chọn vị trí cất cánh cho UAV giá rẻ phù hợp với điều kiện địa hình mỏ. Do vậy, nghiên cứu này sẽ giới thiệu phương pháp xác định vị trí cất cánh phù kỹ thuật máy ảnh sử dụng đi kèm. hợp cho thiết bị bay UAV/RTK, một thiết bị đang được dùng phổ biến trong sản xuất, khi đo vẽ thành lập bản đồ địa hình tỷ lệ lớn cho các mỏ lộ thiên.

\section{Phương pháp xác định vị trí cất cánh cho máy bay}

\subsection{Tính độ phân giải ảnh mặt đất}

Độ phân giải ảnh mặt đất (GSD) cần tính toán để mô hình DSM đạt được độ chính xác phù hợp với tỷ lệ bản đồ cần thành lập về cả thành phần tọa độ mặt bằng $(\mathrm{X}, \mathrm{Y})$ và độ cao $(\mathrm{H})$. Tính toán theo tỷ lệ bản đồ cần thành lập, GSD được xác định có kích thước pixel trong khoảng $(0,05 \div 0,1) \mathrm{M}(\mathrm{mm})$ (Jacobsen, 2005), để đảm bảo đồng thời yếu tố kỹ thuật và kinh tế thì GSD không vượt quá $0.1 \mathrm{M}$ (mm), với $\mathrm{M}$ là mẫu số tỷ lệ bản đồ cần thành lập.

Xét về thành phần sai số trung phương vị trí điểm trên bản đồ cần thành lập, sai số vị trí điểm $m_{p}=(1 \div 2)$ GSD và sai số thành phần độ cao độ chính xác độ cao $m_{H}=(2 \div 3)$ GSD (Trần Trung Anh và nnk., 2019), để đảm bảo đồng thời yếu tố kỹ thuật và kinh tế thì nên chọn:

$$
\begin{aligned}
G S D_{P} & =\frac{m_{p}}{2} \\
G S D_{H} & =\frac{m_{H}}{3}
\end{aligned}
$$

Cần tính toán GSD theo cả hai công thức (1) và (2), GSD được chọn hay GSD cho phép $\left(G S D_{c p}\right)$ sẽ là GSD nhỏ nhất tính được từ hai công thức này vì GSD càng nhỏ độ chính xác càng cao.

\subsection{Tính chiều cao bay chụp}

Chiều cao bay chụp của máy bay được tính là khoảng cách từ điểm máy bay cất cánh đến vị trí máy bay bay chụp ảnh theo dải bay đã thiết kế. Chiều cao này được tính theo công thức sau:

$$
h_{b c}=\frac{W_{i m} \cdot G S D \cdot \ell_{f}}{W_{S} \cdot 100}
$$

Trong đó: $W_{i m}$ - độ rộng tấm ảnh (pixel); GSD - độ phân giải ảnh mặt đất $(\mathrm{cm}) ; \ell_{f}$ - Chiều dài tiêu cự máy ảnh $(\mathrm{mm})$ và $W_{S}$ - độ rộng cảm biến máy ảnh.

Để có được các thông số $W_{i m}, \ell_{f}$ và $W_{S}$ cần biết được loại máy bay sử dụng và thông số 


\subsection{Xác định vị trí cất cánh}

Vị trí máy bay cất cánh phải được chọn tại khu vực bằng phẳng, thông thoáng, ít phương tiện và người qua lại, không dưới đường dây điện.

Xét đến yếu tố vị trí mặt bằng, UAV nên được cất cánh trong khu vực đo vẽ, trong tầm quan sát của người điều khiển để đảm bảo an toàn cho máy bay và tiết kiệm pin (Stöcker và Stöcker, 2017; Vela và nnk., 2018).

Độ cao của điểm UAV cất cánh liên quan đến chiều cao bay chụp $\left(\mathrm{h}_{\mathrm{bc}}\right)$, chiều cao này được tính từ vị trí cất cánh. Mặt khác, $\mathrm{h}_{\mathrm{bc}}$ là một trong các yếu tố quyết định đến độ phân giải ảnh mặt đất GSD và nó được tính toán tự động trên phần mềm bay chụp. Để toàn bộ DSM đạt độ chính xác, cần xác định được $h_{b c}$ sao cho mọi điểm yếu nhất trên DSM có GSD đạt độ chính xác theo yêu cầu của bản đồ cần thành lập.

Trên Hình 1, nếu máy bay cất cánh tại vị trí có độ cao trung bình của mỏ thì độ cao điểm cất cánh được tính theo công thức (4).

$$
H_{F}=\frac{H_{\max }-H_{\min }}{2}
$$

Độ cao bay chụp của máy bay được xác định theo công thức (5).

$$
H_{b c}=H_{F}+h_{b c}
$$

Chiều cao bay chụp tại nơi có địa hình thấp nhất được xác định theo công thức (6).

$$
h_{\text {max }}=H_{b c}-H_{\text {min }}
$$

Độ phân giải không gian mặt đất tại điểm có độ cao thấp nhất trên địa hình được xác định theo công thức (7).

$$
G S D_{y}=\frac{h_{\text {max }} \cdot W_{S} \cdot 100}{W_{i m} \cdot \ell_{f}}
$$

Để đảm bảo độ chính xác bản đồ theo tỷ lệ cần thành lập thì $G S D_{y} \leq G S D_{c p}$ (tính theo tỷ lệ bản đồ cần thành lập mục 2.1).

Ngoài ra, để đảm bảo an toàn cho máy bay, vị trí điểm cất cánh của máy bay phải được chọn sao cho máy bay phải bay cao hơn điểm cao nhất của địa hình, chiều cao bay chụp tối thiểu thỏa mãn điều kiện (8).

$$
h_{b c} \geq\left(H_{\max }-H_{F}\right)+h_{\min }
$$

Chiều cao bay chụp tại nơi có cộ cao lớn nhất trên địa hình $\mathrm{h}_{\min }$ (Hình 1 ) được xác định theo (Aerotas, 2020) là $h_{\min } \geq 15 \mathrm{~m}$ để đảm bảo an toàn cho UAV, và với địa hình có các đỉnh cao $h_{\min } \geq 30$ $\mathrm{m}$ để tránh được sự thiếu hụt về độ phủ trùm ảnh (Hình 2)

\subsection{Chia khu bay theo địa hình}

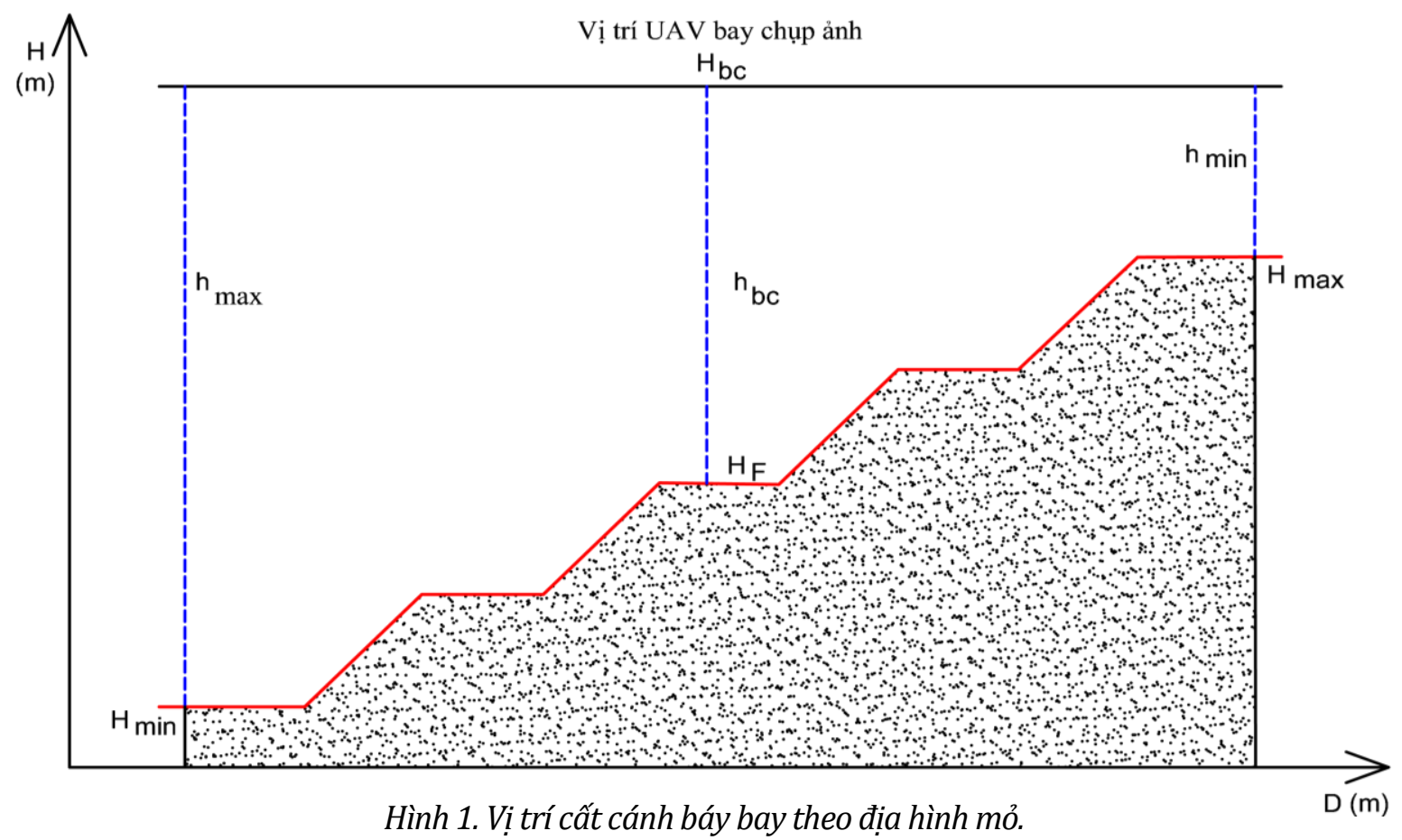


Nếu địa hình có chênh cao lớn, khoảng chênh cao lớn nhất $\left(\Delta h_{\max }\right)$ lớn hơn chiều cao bay chụp cho phép $\left(h_{b c_{c p}}\right)$, thì khu vực đo vẽ cần phải được chia nhỏ thành nhiều khu vực. Tại mỗi khu vực nhỏ cần tính toán để chọn vị trí cất cánh cho máy bay phù hợp với điều kiện địa hình của từng khu.

Số lượng khu vực đo vẽ cần phải chia nhỏ, được tính toán dựa trên chiều cao bay chụp tối đa và chênh cao địa hình lớn nhất của khu vực đo vẽ. Số khu bay được xác định theo công thức (9).

$$
N=\frac{\Delta h_{\max }}{h_{b c_{c p}}}
$$

Trong đó: $h_{b c_{c p}}$ - chiều cao bay chụp cho phép được tính theo công thức (3) với giá trị GSDcp, $\Delta h_{\max }=H_{\max }-H_{\min }$.
Lưu ý $N$ luôn được làm tròn lên.

\section{Thực nghiệm}

\subsection{Khu vực nghiên cứu}

Khu vực có diện tích khoảng 80 ha, tại cánh Đông của mỏ than Cọc Sáu (Hình 3). Khu vực có địa hình tầng bậc phức tạp, chênh cao địa hình lớn nhất theo hướng đường phương của bờ tầng là khoảng $100 \mathrm{~m}$ và theo hướng dốc của bờ tầng là khoảng $300 \mathrm{~m}$.

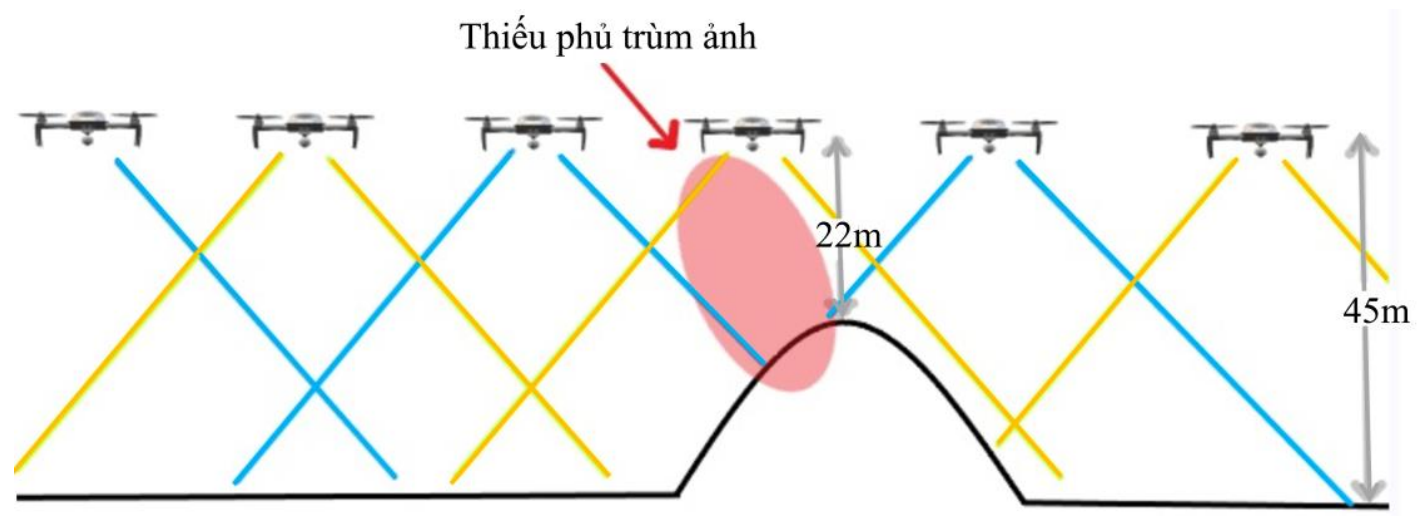

Hình 2. Thiếu độ phủ trùm ảnh do chiều cao bay chụp thấp (Aerotas, 2020)

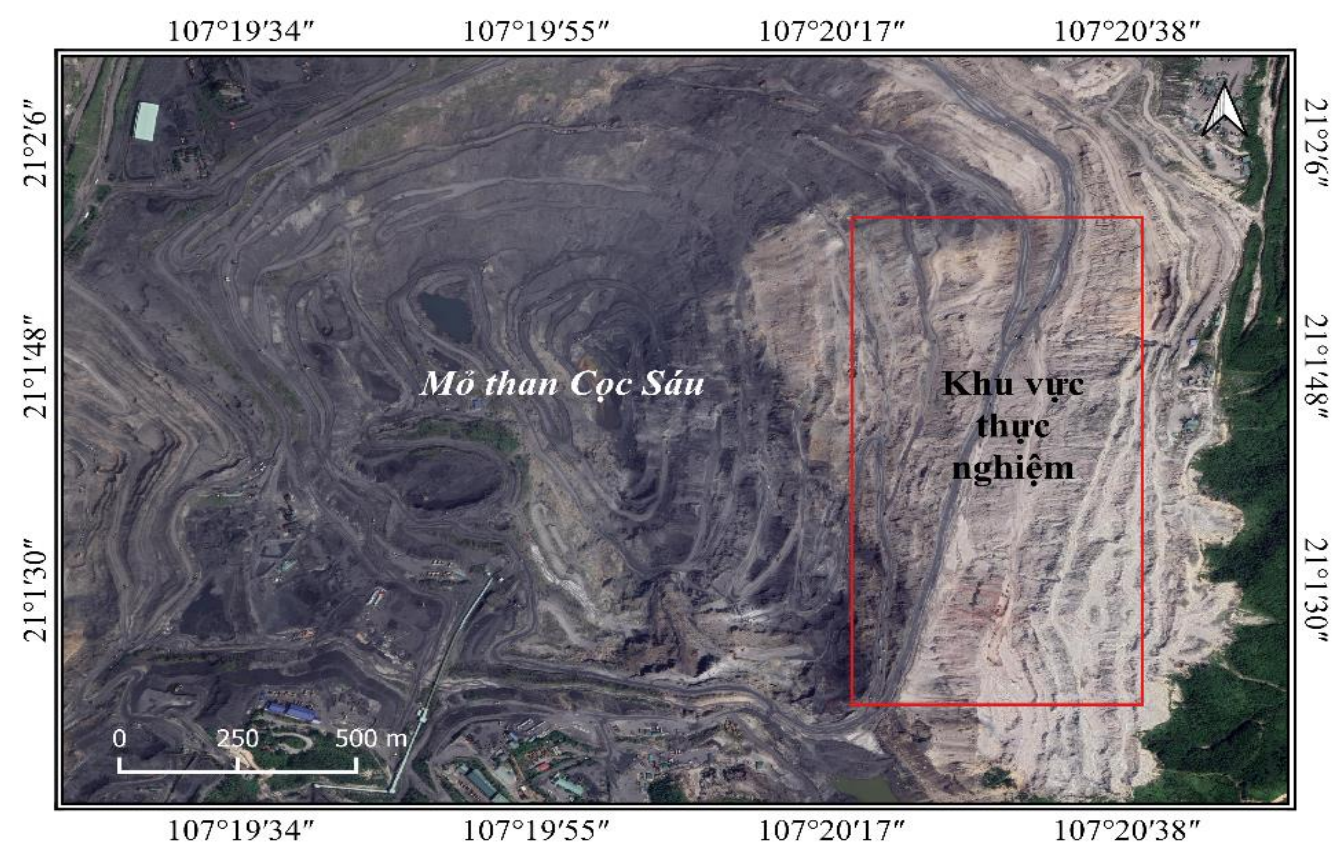

Hình 3. Khu vực nghiên cúu tại mỏ than Cọc Sáu. 


\subsection{Thiết bị sử dụng}

Máy toàn đạc điện tử Topcon ES 105 có độ chính xác đo góc \pm 5 " và độ chính xác đo chiều dài $2 \mathrm{~mm} \pm 2 \mathrm{ppm}$ đã được sử dụng để đo nối tọa độ cho các điểm khống chế ảnh (GCP) (Trắc địa Hoàng Minh, 2020); Máy GNSS Comnav T300 được sử dụng làm trạm cơ sở mặt đất (base), đây là loại máy đo GNSS 2 tần số, độ chính xác định vị mặt bằng và độ cao lần lần lượt là $10 \mathrm{~mm}+0,5$ ppm và $20 \mathrm{~mm} \pm 0,5$ ppm (SinoGNSS,2020). Thiết bị bay không người lái $\mathrm{P} 4 \mathrm{~K}$ được sử dụng để bay chụp địa hình (Hình 4).

P4K được trang bị máy ảnh với độ phân giải $20 \mathrm{Mb} /$ pixel, với chiều dài tiêu cự $\ell_{f}=2,4 \mathrm{~mm}$, độ rộng kích thước sensor $(13,2 \times 8,8)$, kích thước ảnh chụp 4864×3648 (ảnh 4:3) (DJI, 2020). Từ thông số này có thể thấy rằng chiều rộng ảnh là $\mathrm{W}_{\mathrm{im}}=4864$ và kích thước 1 pixel trên sensor là $\mathrm{W}_{\mathrm{S}}$ $=2,7 \mathrm{~mm}$.

\subsection{Xác định vị trí cất cánh cho máy bay}

Tại bể than Quảng Ninh, các mỏ than khai thác lộ thiên được cơ giới hóa toàn bộ, nên việc đo vẽ cập bản đồ địa hình ở mỏ thành lập ở tỷ lệ tỷ lệ 1:1000 với khoảng cao đều đường đồng mức $1 \mathrm{~m}$ (Tập đoàn Công nghiệp Than - Khoáng sản Việt Nam, 2015). Với bản đồ này, sai số vị trí mặt bằng không vượt quá $0.1 \times M=10 \mathrm{~cm}$, về độ cao không vượt quá $1 / 4$ khoảng cao đều là $25 \mathrm{~cm}$.

Để đạt được độ chính xác trên, độ phân giải ảnh mặt đất tối thiểu được tính toán theo công thức (1) và (2) có kết quả lần lượt là $G S D_{P}=5 \mathrm{~cm}$ và $G S D_{H}=12,5 \mathrm{~cm}$. Như vậy, độ phân giải ảnh mặt đất tối thiểu phải đạt được là $G S D_{c p}=5 \mathrm{~cm}$.

Chiều cao bay chụp tối đa:

$$
h_{b c}=\frac{W_{i m} \cdot G S D_{c p} \cdot \ell_{f}}{W_{S} \cdot 100} \approx 216 \mathrm{~m}
$$

Vậy khi bay chụp khoảng cách thẳng đứng từ máy bay tới địa hình mặt đất phải $\leq 216 \mathrm{~m}$.

Khảo sát địa hình khu vực nghiên cứu cho thấy chênh cao địa hình lớn nhất theo hướng đường phương của bờ tầng là $70 \mathrm{~m}$ và theo hướng đường dốc là $300 \mathrm{~m}$ (từ - $60 \mathrm{~m}$ tới $+240 \mathrm{~m}$ ). Do vậy, cần quan tâm chọn vị trí cất cánh cho máy bay sao cho phù hợp với chênh cao theo hướng dốc để đảm bảo độ chính xác GSD và an toàn cho máy bay.

Từ mặt cắt trên Hình 5 , nếu máy bay cất cánh tại vị trí có độ cao trung bình $H_{F}=+90 \mathrm{~m}$, để đảm bảo an toàn cho máy bay chiều cao bay tối thiểu được tính theo công thức (8) là $165 \mathrm{~m}$ (với $H_{\max }=$ $240 \mathrm{~m}, H_{F}=90 \mathrm{~m}, h_{\min }=15 \mathrm{~m}$ ). vậy, độ cao bay chụp tối thiểu của máy bay sẽ là $H_{b c}=255 \mathrm{~m}$. Chiều cao bay chụp lớn nhất tại địa hình $h_{\max }=H_{b c}-H_{\min }=255$ $+60=315$ m, vượt qua chiều cao bay chụp tối đa đã tính ở trên (216 m). Do vậy, cần chia khu vực đo vẽ thành các khu nhỏ. Với chênh cao địa hình lớn nhất của khu đo khoảng $300 \mathrm{~m}$, chiều cao bay chụp cho phép tối đa $216 \mathrm{~m}$, thay vào công thức (9), tính được $N=1,39$. Do vậy, cần chia khu vực địa hình làm hai mức bay chụp, tại mỗi mức bay chụp chọn cất cánh ở vị trí có độ cao trung bình tương ứng là $+50 \mathrm{~m}$ và $+160 \mathrm{~m}$, chiều cao bay chụp sẽ là $100 \mathrm{~m}$ đảm bảo cao hơn điểm cao nhất của địa hình $(>20 \mathrm{~m})$. Vị trí điểm sâu nhất của địa hình có chiều cao bay chụp đều là $210 \mathrm{~m}$ nhỏ hơn chiều cao bay chụp tối đa (216 m).

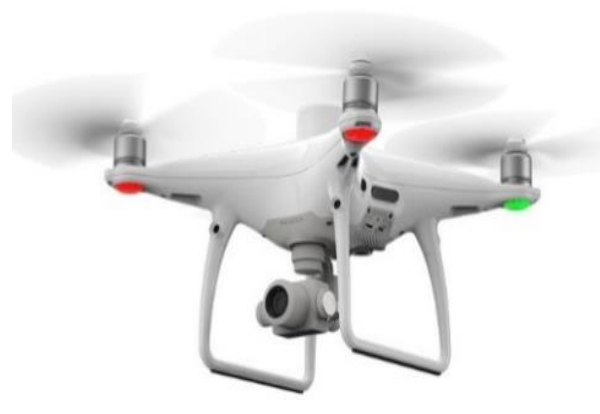

(a)

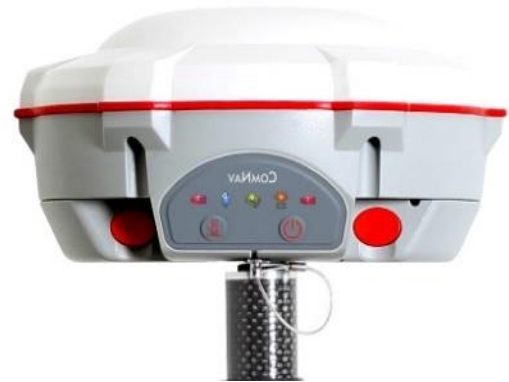

(b)

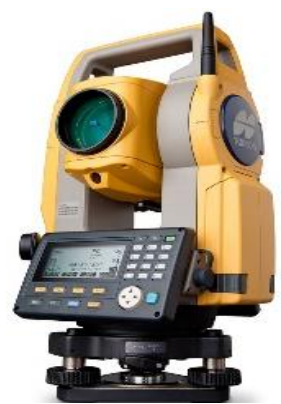

(c)

Hình 4. Thiết bị sử dụng.

a. Thiết bị bay Phantom 4 RTK; b. Máy thu GNSS Comnav T300; c. Máy toàn đạc điện tử Topcon ES 105. 


\subsection{Bay chụp ảnh thục nghiệm}

Tổng số ca bay theo thiết kế làm 4 ca bay (Hình $5 \mathrm{a}$ ) trong đó ca bay 1 và 2 được thực hiện cho vùng địa hình có độ cao thấp (độ cao dưới 125 $\mathrm{m}$ ) và máy bay sẽ cất cánh tại vị trí $T_{1}$ (ở mức độ cao $+50 \mathrm{~m}$ ), hai ca 3 và 4 bay chụp vùng địa hình còn lại, máy bay sẽ cất cánh tại tại vị trí $T_{2}$ (ở mức độ cao $+160 \mathrm{~m}$ ). Độ phủ trùm ảnh là $75 \%$ theo cả chiều dọc và chiều ngang, chiều cao bay chụp 100 m, tổng số ảnh bay chụp được là 808 ảnh. Tâm ảnh được định vị theo phương thức đo động xử lý sau GNSS/PPK, máy thu cố định (base) được đặt tại điểm mốc giải tích 1 của mỏ (Hình 6a).

\subsection{Thành lập DSM}

Tọa độ tâm chụp của các ảnh khi bay chụp bằng $\mathrm{P} 4 \mathrm{~K}$ theo phương phức đo động thời gian thực cần được xử lý, tính toán tọa độ tâm chụp chính xác trước khi sử dụng.

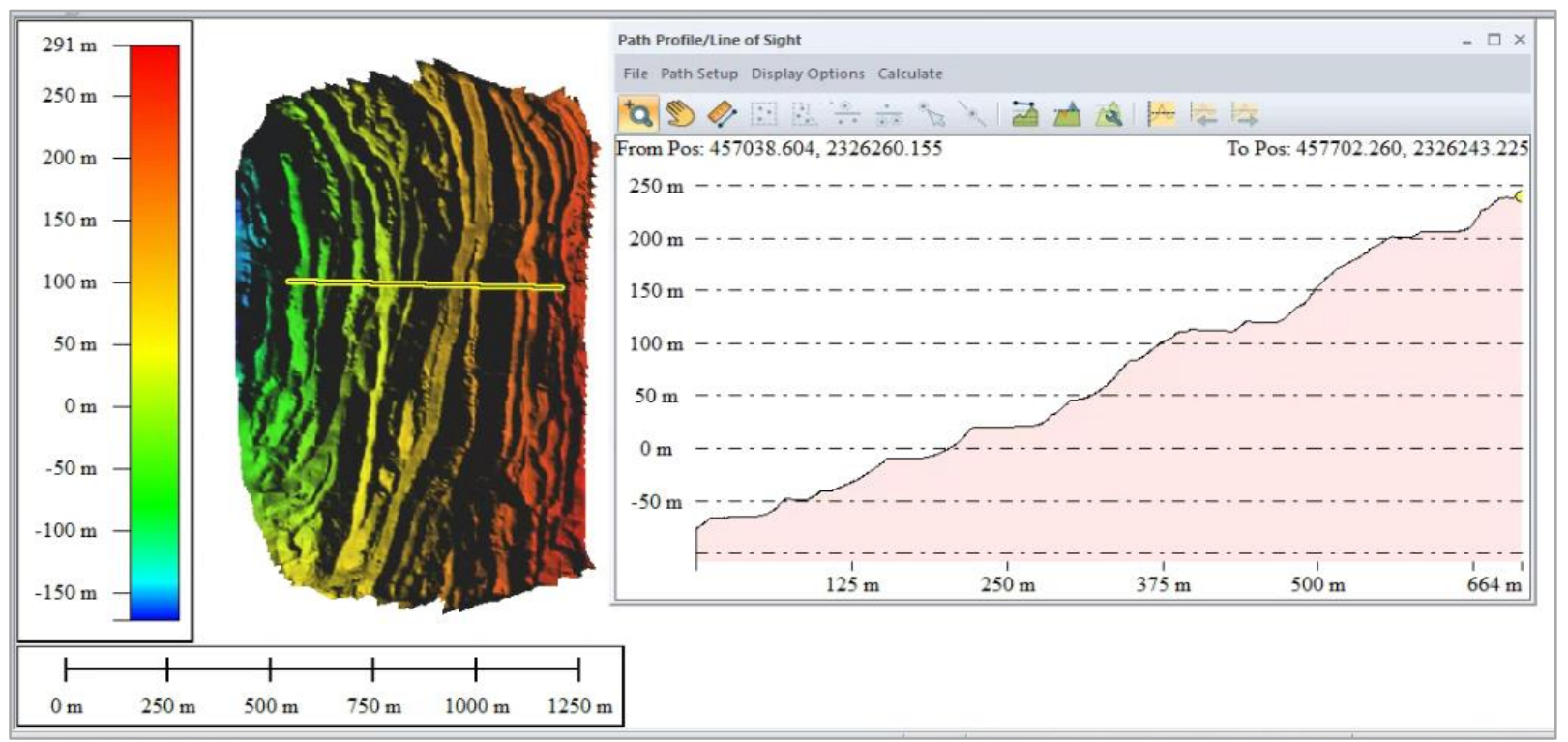

Hình 5. Mặt cắt địa hình theo dốc bò̀ tầng khu vực nghiên cứu tại mỏ than Cọc Sáu.

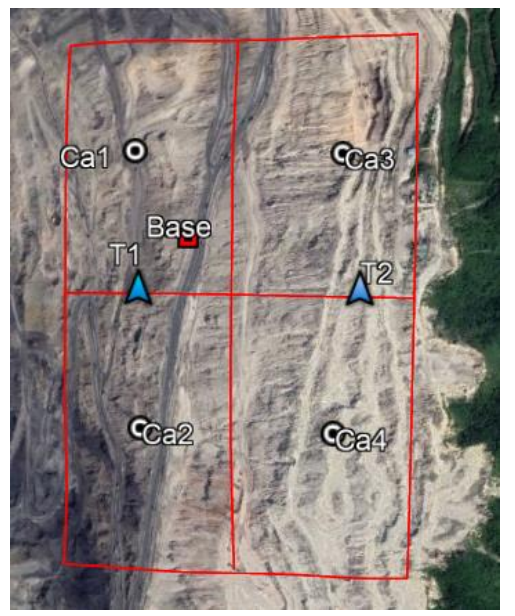

(a)

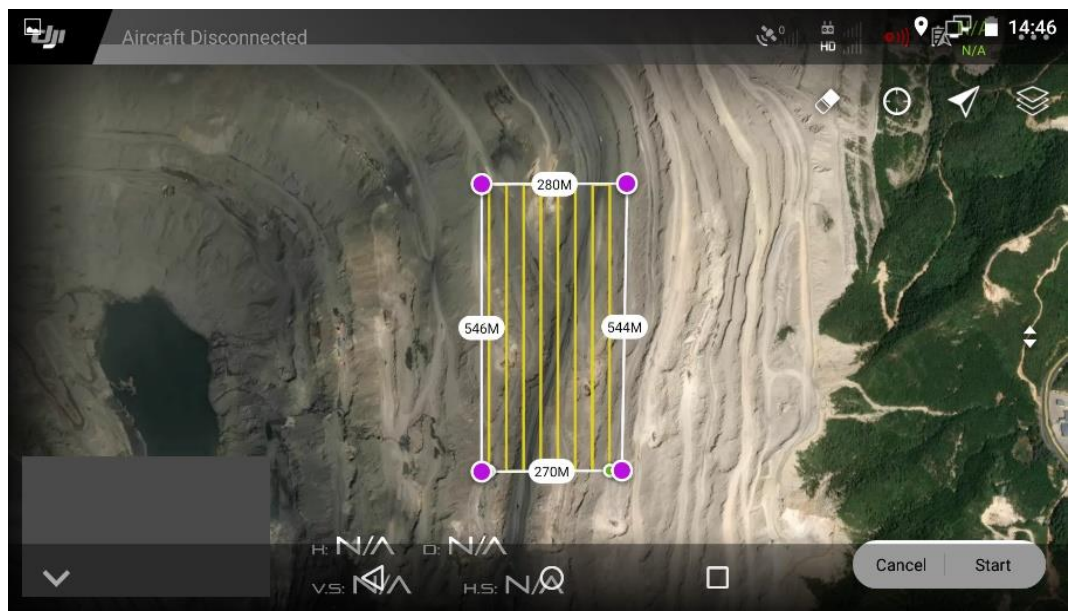

(b)

Hình 6. Kế hoạch bay chụp

a. So đồ ca bay trên Google Earth; b. Thiết kế ca bay trên phần mềm điều khiển DJI GS RTK.

Tọa độ tâm chụp của các ảnh được tính toán từ các file dữ liệu rinex trong máy base và máy bay, file lịch vệ tinh chính xác được cung cấp bở Nasa. Việc tính toán này được thực hiện trên các 
phần mềm RTKlib và Aerotas P4RTK $\mathrm{PPK}$ Adjustments. Quá trình ghép tọa độ tâm chụp mới cho các ảnh, xử lý ảnh và thành lập DSM thực hiện trên phần mềm Agisoft Metashape Professional.
DSM được thành lập với 3 trường hợp: (1) PPK chỉ sử dụng ảnh UAV/RTK, (2) PPK + 1 GCP và (3) PPK+ 2 GCP, và được đánh giá độ chính xác thông qua các điểm khống chế mặt đất đã thành lập.

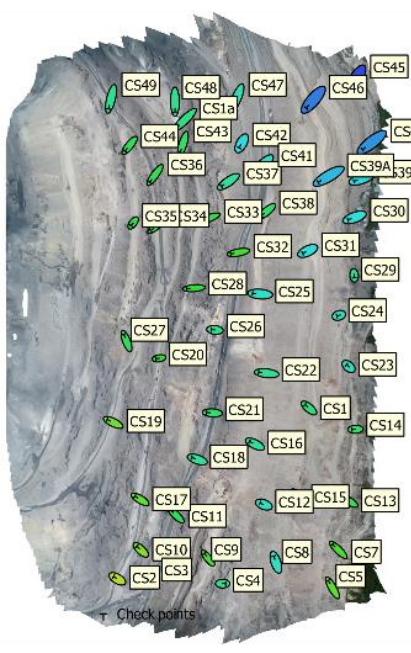

(a)

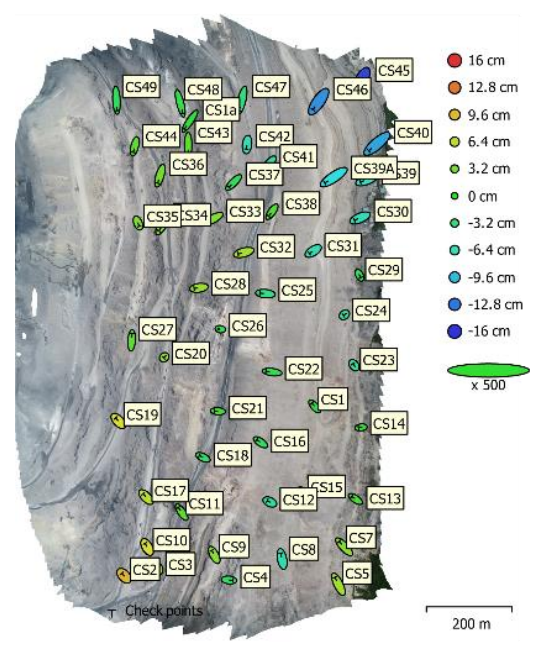

(b)

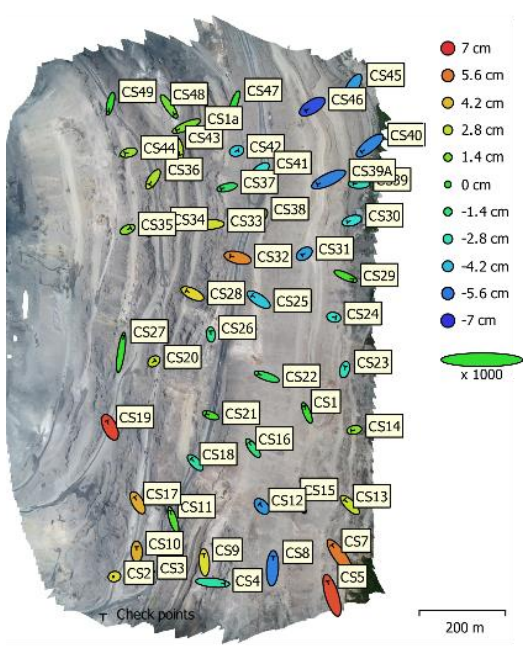

(c)

Hình 7. Vị trí các điểm khống chế ảnh mặt đất và elipsai số (tù̀ Agisoft Metashape).

a. PPK; b. $P P K+1 G C P ; c . P P K+2 G C P$.

Bảng 1. Sai số vị trí 5 điểm yếu nhất trên các mô hình DSM

\begin{tabular}{|c|c|c|c|c|}
\hline \multirow{2}{*}{ Điểm } & \multicolumn{5}{|c|}{ Sai số $(\mathrm{cm})$} \\
\cline { 2 - 5 } & $\Delta X$ & $\Delta Y$ & $\Delta X Y$ & $\Delta H$ \\
\hline PPK & $-2,3$ & $-4,3$ & 4,8 & $-10,1$ \\
\hline CS42 & $-7,3$ & $-2,5$ & 7,7 & $-10,4$ \\
\hline CS39 & $-9,7$ & $-5,6$ & 11,3 & $-12,2$ \\
\hline CS39A & $-8,7$ & $-6,8$ & 11,1 & $-14,4$ \\
\hline CS40 & $-6,7$ & $-7,7$ & 10,2 & $-15,6$ \\
\hline CS46 & $-5,4$ & $-9,1$ & 10,6 & $-18,7$ \\
\hline CS45 & $-5,5$ & $-1,9$ & 5,9 & $-5,6$ \\
\hline PPK + 1 GCP & $-7,5$ & $-5,0$ & 9,0 & $-6,9$ \\
\hline CS39 & $-7,0$ & $-6,0$ & 9,2 & $-9,1$ \\
\hline CS39A & $-4,6$ & $-6,8$ & 8,2 & $-10,1$ \\
\hline CS40 & $-3,7$ & $-8,1$ & 8,9 & $-13,3$ \\
\hline CS46 & $-1,1$ & 1,2 & 1,6 & $-5,0$ \\
\hline CS45 & $-3,9$ & $-3,1$ & 5,0 & $-5,3$ \\
\hline PPK + 2 GCP & $-5,2$ & $-2,4$ & 5,8 & $-5,3$ \\
\hline CS12 & 0,3 & 5,5 & 5,5 & $-5,4$ \\
\hline CS40 & $-3,2$ & $-1,9$ & 3,7 & $-6,4$ \\
\hline CS39A & \multicolumn{5}{|l|}{}
\end{tabular}




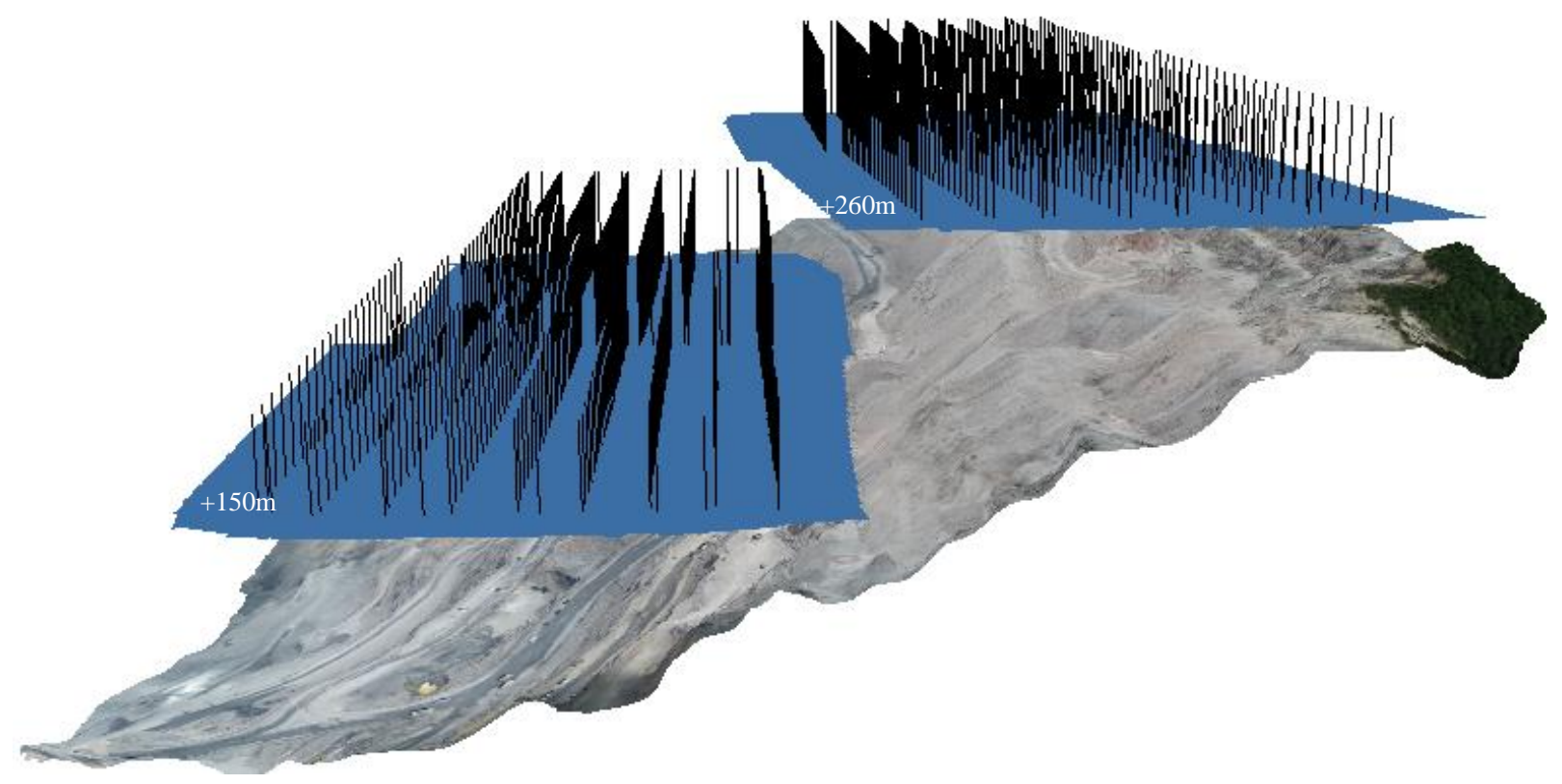

Hình 8. Vị trí các ảnh được chụp khi máy bay cất cánh tại vị trí $T_{1}$ và $T_{2}$

\section{Kết quả và thảo luận}

Mặt khác, do bay chụp ở 2 độ cao khác nhau, đã giảm được sai số tại các điểm ở các khu vực có độ cao nhỏ nhất trên mô hình. Elip sai số của các điểm kiểm tra trên Hình 7 thể hiện sự phân bố đều trên cả khu vực nghiên cứu về sai số, không tập trung vào khu vực có độ sâu lớn nhất. Cũng từ Hình 7 thấy rằng, nếu không sử dụng điểm khống chế ảnh thì sai số vị trí điểm kiểm tra trên DSM có giá trị lớn nhất đề ti mét, sai số này được cải thiện khi dùng 01 điểm khống chế ảnh và đạt giá trị $\mathrm{cm}$ khi dùng 02 điểm khống chế ảnh.

Nếu xét theo sai số thành phần tọa độ được thể hiện trên Bảng 1 thì sai số vị trí mặt bằng $(X$, Y) của các DSM thuộc cả 3 trường hợp đều có giá trị nhỏ, giá trị lớn nhất là $11 \mathrm{~cm}$, đạt độ chính xác thành lập bản đồ địa hình tỷ lệ lớn theo qui phạm trắc địa mỏ. Tuy nhiên, thành phần độ cao $(\mathrm{H})$ có sai số lớn hơn, sai số độ cao đạt đề xi mét khi không sử dụng bất kỳ điểm GCP nào, đạt đến $\mathrm{cm}$ khi sử dụng 02 điểm GCP (Bảng 1).

\section{Kết luận}

Trong nghiên cứu này, với mục đích xác định được vị trí cất cánh phù hợp cho thiết bị bay UAV tích hợp GNSS động khi đo vẽ thành lập bản đồ địa hình tỷ lệ lớn cho các mỏ lộ thiên. Kết quả nghiên cứu cho thấy:
- Cần khảo sát địa hình mỏ lộ thiên, thiết kế vị trí cất cánh cho máy bay sao cho GSD của điểm thấp nhất trên mô hình đạt độ chính xác theo yêu cầu của bản đồ cần thành lập;

- Khi khu vực đo vẽ có chênh cao địa hình lớn hơn chiều cao bay chụp cho phép. Cần phải chia khu vực bay chụp thành nhiều khu nhỏ dựa trên chênh cao lớn nhất của địa hình và chiều cao bay chụp cho phép. Trong mỗi khu vực đã được chia nhỏ, nên chọn vị trí cất cánh tại nơi có độ cao trung bình; Về mặt bằng, điểm cất cánh nên ở trong phạm vi khu bay chụp nhằm đảm bảo yếu tố an toàn và tiết kiệm pin.

- Sử dụng ảnh bay chụp từ Phantom 4 RTK kết hợp với 02 điểm khống chế ảnh mặt đất để DSM có thể đạt độ chính xác cm.

\section{Lời cảm ơn}

Bài báo này là sản phẩm khoa học của đề tài mã số T20 - 06, nhóm tác giả chân thành cảm ơn Trường đại học Mỏ - Địa chất đã hỗ trợ kinh phí, cảm ơn Cồng ty CP than Cọc Sáu đã hồ trợ trong quá trình đo đạc thực nghiệm tại mỏ.

\section{Tài liệu tham khảo}

Aerotas.(2020). https://www.aerotas.com/ choosing - flight - altitude.

Bùi Tiến Diệu, Nguyễn Cẩm Vân, Hoàng Mạnh Hùng, Đồng Bích Phương, Nhữ Việt Hà, Trần 
Trung Anh, Nguyễn Quang Minh (2016). Xây dựng mô hình số bề mặt và bản đồ trực ảnh sử dụng công nghệ đo ảnh máy bay không người lái. Hội nghị khoa học Đo đạc bản đồ với ứng phó biển đổi khí hậu, Hà Nội.

Dieu Tien Bui, Nguyen Quoc Long, Bui Xuan Nam, Nguyen Viet Nghia, Pham Van Chung, Le Van Canh, Bjørn Kristoffersen, (2017). Lightweight Unmanned Aerial Vehicle and Structure - from - Motion Photogrammetry for Generating Digital Surface Model for Open - Pit Coal Mine Area and Its Accuracy Assessment. International Conference on Geo - Spatial Technologies and Earth Resources, 17 - 33.

Dinkov, D., \& Kitev, A. (2020). Advantages, disadvantages and applicability of GNSS post processing kinematic (PPK) method for direct georeferencing of uav images. 8th International Conference on Cartography and GIS,1,747749.

DJI. (2020). Phantom 4 RTK Visionary Intelligence. https://www.dji.com/phantom 4 - rtk.

He, J., Li, Y., \& Zhang, K., (2012). Research of UAV Flight Planning Parameters. Positioning, 03, 43 - 45. doi:10.4236/pos.2012.34006.

Jacobsen, K., (2005). Photogrammetry and geoinformation trends in large scale mapping.

Lê Văn Cảnh, Cao Xuân Cường, Lê Hồng Việt, \& Đinh Tiến. (2020). Ứng dụng công nghệ bay không người lái (UAV) trong đo đạc phục vụ công tác tính trũ lượng các mỏ đá tại Việt Nam. Tạp chí Khoa học kỹ thuật Mỏ - Địa chất, 61, 21 - 30. doi:10.46326/JMES.2020.61(1).03

Lee, S., \& Choi, Y., (2015). On - site demonstration of topographic surveying techniques at open pit mines using a fixed - wing unmanned aerial vehicle (drone). Tunnel and Underground Space, 25(6), 527 - 533.

Nguyễn Đình Bé, Võ Chí Mỹ, Nguyễn Xuân Thụy.
(1998). Trắc địa mỏ. Nhà xuất bản Giao thông Vận tải.

Nguyen Quoc Long, Bui Xuan Nam, Cao Xuan Cuong, \& Le Van Canh. (2019). An approach of mapping quarries in Vietnam using low - cost Unmanned Aerial Vehicles. International Journal of Sustainable Development, 11(2),199 210. doi:10.21177/1998 - 4502 - 2019 - 11 - 2 $-199-210$.

Nguyễn Quốc Long, \& Lê Văn Cảnh. (2020). Khả năng ứng dụng thiết bị bay không người lái (UAV) kinh phí thấp để đo vẽ kiểm kê trữ lượng khoáng sản mỏ lộ thiên. Công nghiệp mỏ 29(2), $79-85$.

SinoGNSS, (2020). T300 Plus GNSS Solution. http://www.comnavtech.com/t300p_solution .html.

Stöcker, C., Bennett, R., Nex, F., Gerke, M., \& Zevenbergen, J., (2017). Review of the Current State of UAV Regulations. Remote Sensing 9(5), 459.

TCVN 10673:2015 (2015). Tập đoàn Công nghiệp Than - Khoáng sản Việt Nam. Tập 1. Bộ khoa học và Công nghệ.

Trắc địa Hoàng Minh. (2020). Máy toàn đạc điện tử Topcon ES 105, http://homicom.vn/may toan - dac - topcon - es105.html.

Trần Trung Anh, Dương Thế Anh, Phạm Viết Kiên, \& Lê Như Ngọc. (2019). Kết hợp công nghệ UAV, RTK và SES trong thành lập bản đồ địa hình tỷ lệ lớn vùng rùng ngập mặn ven biển. Hội nghị toàn quốc khoa học trái đất và tài nguyên với phát triển bền vững - ERSD2019.

Võ Chí Mỹ. (2016). Trắc địa mỏ. Nhà xuất bản Khoa học Tự nhiên và Công nghệ.

Vela, A. E., Ferreira, L., \& Babin, T., (2018). $A$ Safety Analysis of UAV Mapping Operations. Paper presented at the 2018 IEEE/AIAA 37th Digital Avionics Systems Conference (DASC). 